\title{
Integration of Georeferencing, Habitat, Sampling, and Genetic Data for Documentation of Wild Plant Genetic Resources
}

\author{
Gayle M. Volk ${ }^{1}$ and Christopher M. Richards \\ National Center for Genetic Resources Preservation, U.S. Department of Agriculture, 1111 S. Mason Street, \\ Fort Collins, CO 80521
}

Additional index words. core collection, database, genebank, genomic, plant breeding

\begin{abstract}
Plant genetic resource collections provide novel materials to the breeding and research communities. Crop wild relatives may harbor completely novel forms of allelic variation for biotic and abiotic resistance as well as masked genes for improved quality and production. This variation has been shaped by the environment from which the plant materials were collected. With detailed original source information, genetic assessments of germplasm collections can go beyond the basic measurements of collection diversity and breeding for simple traits to assessments of natural variation in environmental contexts. Availability of detailed documentation of passport, phenotypic, and genetic data increases the value of all genebank accessions. Inclusion of georeferenced sources, habitats, and sampling data in collection databases facilitates interpretation of genetic data for genebank accessions with wild origins.
\end{abstract}

Wild plant genetic resources within genebanks provide researchers with access to valuable genotypic and phenotypic diversity. In particular, crop wild relatives are emerging as very important genetic resources that provide breeders and geneticists with desirable alleles and gene combinations that may provide sources of improved quality, yield as well as disease and pest resistance to cultivated crops (Hajjar and Hodgkin, 2007). Crop wild relatives may harbor completely novel forms of allelic variation for biotic and abiotic resistance as well as masked genes for improved quality and production. Although crops vary in the extent of domestication that has occurred as a result of selection by or coevolution with humans, there are examples in which rare alleles were lost in the domestication process (Hyten et al., 2006).

Wild germplasm collections contain variation that is shaped by both demographic history and population structure. The particular worth of these wild collections is that they represent both a living sample useful for breeding and, in contrast to cultivated accessions, they are associated with a specific collection point and this place sets the accession in an ecological context. Collections of wild species held in gene banks around the world operate therefore at an interface between evolutionary biology and applied conservation biology (Schoen and Brown, 2001; Walters et al., 2008). In theory, plant germplasm collections offer a tremendous potential for building prebreeding

Received for publication 8 Apr. 2011. Accepted for publication 16 June 2011.

We thank Briana Gross for providing a manuscript review.

Mention of trade names or commercial products in this publication is solely for the purpose of providing specific information and does not imply recommendation or endorsement by the U.S. Department of Agriculture. USDA is an equal opportunity provider and employer.

${ }^{1}$ To whom reprint requests should be addressed; e-mail Gayle.Volk@ars.usda.gov. programs that could help locate and ultimately exploit this variation in agricultural breeding programs. In practice, systematic exploitation of natural variation from wild relatives is a major investment of time and effort.

Plant breeders have long used crosses to manipulate plant phenotypes. By making wide crosses they can often open new combinations with useful characters. Transgressive variation, for example, is the phenomenon of hybrids with parents of different origins (ecotypes within a species) displaying unusual phenotypes that are often useful for understanding the genetic basis of agricultural traits (Gur and Zamir, 2004; Tanksley and McCouch, 1997). What has been demonstrated using both traditional breeding and molecular approaches is that phenotype is not a good predictor of genetic potential and that the epistatic interaction of loci from different lineages can result in novel and potentially valuable phenotypes. Segregating populations derived from these crosses can be recurrently backcrossed to a crop parent to introduce wild alleles into a domesticated background. The methods have not only been critical in introducing traits from the wild into agricultural crops, but they have provided research materials necessary to identify the genetic basis of these traits using genomic methods. Model systems such as rice, tomato, and Arabidopsis have demonstrated significant genetic potential of exploiting transgressive variation in breeding programs (McCouch, 2004).

A complementary approach to creating pre-breeding populations is leveraging understanding of gene function to conduct surveys of naturally occurring allelic variants at genes of agronomic importance (e.g., Johal et al., 2008). With continued improvements in sequencing technologies, bioinformatic capacity, and analytical methods, genomic analysis is moving away from model systems and is increasingly applied to crop wild relatives in association studies and allele mining (Kumar et al., 2010). Aided by functional characterization using mutational screens, quantitative trait loci mapping, and whole genome asso- ciation studies, loci of agricultural importance have been identified and surveys of alleles at these loci have focused on naturally occurring variants for use in crop improvement (Bishop et al., 1996; Chapman et al., 2008; GonzálezMartínez et al., 2007; Vigouroux et al., 2002; Xiao et al., 1996). Novel alleles recovered at these loci can be integrated into crop breeding programs using conventional or molecular approaches and could be used to combat disease (Bhullar et al., 2009; Caicedo, 2008; Kaur et al., 2008; Wang et al., 2006), promote yield increases, produce better storage and nutritional properties, or improve stress tolerance (Latha et al., 2004). By dissecting how allelic variation controls the amplitude of a trait value, not only are breeders able to identify what allele to cross into cultivars, but geneticists can now describe gene genealogies for these loci and begin to understand the evolutionary process that gave rise to this variation.

Putting functional variation into an ecological context greatly enhances our understanding of the process that leads to adaptation. This may be particularly relevant to the development and identification of germplasm valuable for mitigation of global climate change. Adaptive differentiation forms the basis of biodiversity and serves as a major focus of evolutionary biology. The details focus not only on the specific genetic architecture and functional characteristics of specific loci, but also how variation at these genes arise and are maintained under natural selection.

Until recently the genetic mechanisms of specific gene function and the evolutionary and population genetic processes that shape diversity have been treated separately. Bridging this gap and integrating genetic, spatial, and historical context has been the focus of ecological genetics (Antonovics, 1976) and more recently population and ecological genomics (Black et al., 2001; Luikart et al., 2003). A major driver of this interaction is the rapid advances in DNA sequencing technology (Mardis, 2008). The ability for individual laboratories to create genomic resources and the availability of annotated and mapped sequences, a wide 
range of model organisms has created a vital opportunity to explore the genetic mechanisms of adaptation on a fundamentally new way (Stapley et al., 2010).

Explicit sample information is critical for analyses that seek to identify adaptive variation in wild germplasm. Global standards for data documentation are critical to bridge the gap between sample context and sample genetics. Accession documentation can include, but is not limited to, passport, phenotypic, genotypic, and even genomic data. Access to organized, digitized, parsed, queriable, and complete information increases the number of requests for that germplasm (Day Rubenstein et al., 2006). Basic passport, geospatial, habitat, and sampling data fields place ex situ samples in their environmental context.

\section{BASIC PASSPORT INFORMATION}

Global standards for documentation of biodiversity have been established. The Global Biodiversity Information Facility (GBIF) serves as a data portal for accessing primary biodiversity data from natural history collections and also observational data (Edwards et al., 2000). These data include specimen occurrences, records of plants and animals in nature as well as curation institutions and projects. Taxonomic Data Working Group (TDWG) is the recommended format for GBIF data submission (Kelling, 2008). The standards were specifically developed to allow institutions and organizations to share compatible biorepository information about their collections (Kennedy et al., 2006). TDWG standards describe the collection source, taxonomy, geographical source description, biological elements, and reference elements (Taxonomic Data Working Group, 2011). These classification categories are described by the Darwin Core data standards to present a vocabulary for occurrence and related spatiotemporal information about accessions (Darwin Core Task Group, 2011). The Darwin Core standards have been widely adopted by national and international databases that compile information about biological collections.

Because standards for passport data are intended to increase database-sharing among communities, basic inventory information is required. The Darwin Core fields include, but are not limited to, categories that provide institution codes and sample identification, taxonomic classification, and basic source locality descriptors to describe geographical references (Table 1; Darwin Core Task Group, 2011). Darwin Core data for many collections are accessible through the GBIF portal, which facilitates searches for plant, animal, and microbial collections and genetic resources at the taxonomic, geographic, and institutional levels (Edwards et al., 2000). Basic occurrence information can be assessed at a species level using country of origin and taxonomic data.

\section{GEOSPATIAL EXTENSION}

Darwin Core standards provide locality data based on political boundaries and locality names. As boundaries and cities change over time, identification of source locations becomes difficult to ascertain. Specific georeferencing descriptors based on precise latitude and longitude measurements remain consistent over time; thus, a set of geospatial descriptor extensions (Darwin Core Geospatial Extension) provides a mechanism to document the specific georeferencing data available for accessions (Table 1). The descriptors also identify the uncertainty of the documented data points. Data mining tools have been built for retrospective georeferencing, assigning latitude and longitude data to locality-based historical records (Hill et al., 2009). GIS tools can also be used to confirm georeferenced coordinates within genebank databases (Hijmans et al., 1999).

Geographical data, in addition to genetic data, provide opportunities for collection curators to evaluate diversity for a number of practical applications such as coordinating authenticity of accessions from collections within the country of origin, identification of duplicates, planning explorations to find novel germplasm in the wild, identification of core or reference collections, and determining potential sources of new alleles based on the species' original habitat (Greene and Pederson, 1996; Holbrook et al., 1993; Khoury et al., 2010; Ramírez-Villegas et al., 2010; Williams, 2005). In some cases, localized alleles may have specific adaptations and these novel alleles or allelic combinations can be effectively captured when specific geographies are targeted (Schoen and Brown, 1993). Georeferencing inferences are limited by the lack of documentation for the originally collected plant materials. In many cases, single or few accessions serve as static representations of a region.

\section{HABITAT DESCRIPTION}

Taxonomic, locality, and georeferencing data facilitate collection characterization based on the materials in the ex situ collection but provide limited information about what is going on in nature. Thus, ex situ samples taken from the wild are static elements used for coarse grain inference. As we start to investigate adaptive traits for agriculture that arise from multiple genes, the complexity of the analysis increases and the associated data collected at the time of sampling becomes more critical. The convergence of genomic, sampling, and georeferencing data allow for the estimation of genetic discontinuities, adaptations, and speciation events. The processes of species expansion, differentiation, and adaptation can be inferred when genetic and ecological data are interpreted. Habitat descriptions are needed to estimate the cause of genetic discontinuities. These help to explain the pattern of genetic diversity across the landscape. Knowledge of landscape genetics is crucial for managing the diversity of threatened and endangered populations (Manel et al., 2003).

Well-documented habitat descriptions are useful for correlating genetic differentiation estimates with potential adaptation. These data
Table 1. Selected Darwin core and geospatial extension fields as reported by the Taxonomic Data Working Group (2011).

Inventory

InstitutionCode

CollectionCode

CatalogNumber

Taxonomy

HigherTaxon

Kingdom

Phylum

Class

Order

Family

Genus

Specific Epithet

Infraspecific Rank

Infraspecific Epithet

Locality

Continent

Country

State/Province

County

Locality

Elevation

Collection Event

Date

Collector

Collecting Method

Geospatial Extension

Latitude

Longitude

Geodetic datum

Descriptive fields

can also inform about the history of populations. From a biogeographic data standpoint, estimates of population history are important because they allow for the identification of management units for in situ conservation and provide focus areas for allele mining. With georeferencing data, some habitat data can be retrieved from international databases such as WorldClim (Hijmans et al., 2005).

An informal survey of 23 collection databases revealed habitat fields that are frequently included for passport data documentation. It also revealed that there is a considerable lack of standardization among databases for habitat and sampling documentation. Common habitat-type fields include those relating to physical properties of the collection site and site ownership (Table 2). These descriptors aid in determining whether the collected sample may be of interest to the user community (Steiner and Greene, 1996). They may also describe critical species habitats, information valuable for the conservationists.

\section{SAMPLING DOCUMENTATION}

Samples need to be explicitly defined to accomodate assumptions used in genetic analyses. For example, samples can represent bulked populations, a defined matrilineal family, or an individual. Genetic data must be placed within a context of the sampling structure. Data users need to know if seed samples are homogeneous or heterogeneous. Do 
Table 2. Data fields suggested for documentation of ecogeographic and sampling data for wild plant genetic resources. ${ }^{\mathrm{z}}$

\begin{tabular}{ll}
\hline Suggested habitat descriptors & \multicolumn{1}{c}{ Example categories for habitat descriptors } \\
\hline Land type & Public, private, roadside \\
Land owner & Federal, state, local government, private \\
Soil texture (FAO) & Clay, loam, silt, sand, etc. \\
Soil moisture regime (FAO) & Dry, slightly moist, moist, wet \\
Soil pH & Acid, neutral, alkaline \\
Slope & In degrees \\
Aspect & N, NE, E, SE, S, SW, W, NW \\
Light exposure & Sun, semishade, shade \\
Land use (FAO) & Industrial, recreational, agriculture, forest, residential, etc. \\
Habitat category (IUCN) & Forest, savanna, shrubland, grassland, wetland, rocky, \\
& desert, coastline \\
Site narrative & Text describing site \\
Image of site & Image \\
Anthropogenic disturbance & Cultivated, grazed, mowed, logged, roadway \\
Threat category (IUCN) & None, human induced loss, invasive species, \\
& pollution, natural disaster, etc. \\
Associated plant species & Text \\
& \\
Suggested sampling descriptors & \multicolumn{1}{c}{ Example categories for sampling fields } \\
\hline Plot description & Plot shape, size, and placement \\
Distribution pattern & Solitary, patchy, scattered, solid cover \\
Population size & Number or range \\
Age distribution & Percentage of juvenile and mature individuals \\
No. fruits per individual & Estimated number \\
No. seeds per fruit & Estimated number \\
Phenology at sampling time & Flowers, green fruits, ripe fruits, etc. \\
Collection method & Text \\
Sampling selection or bias & Random, systematic, selected \\
Quantity collected & Number or range \\
Units collected & Count, grams, mg \\
Collected form & Budwood, bulb, cutting, DNA, leaves, plant, seed, tuber, etc. \\
No. plant sampled & Number or range \\
Seed collection source & Plant, ground, both \\
No. seeds per individual & Estimated number \\
Harvest time & Early, mid, late season \\
Improvement status & Clone, cultivar, cultivated, genetic, landrace, \\
& rootstock, wild, uncertain \\
\hline
\end{tabular}

${ }^{\mathrm{z}}$ Examples of habitat and sampling fields are selected from fields used by existing plant collection databases.

individuals within seedlots share common parentage? Are individuals within field collections related through maternal lineages? Population information such as spatial distribution, plant age, possibilities for hybridization, and how individuals in the wild were selected for sampling is important. These data allow for the identification of hierarchical estimates of genetic structure, immigrants, and long-distance gene flow. These data are also critical if ex situ materials are to eventually be reintroduced into the wild (Schoen and Brown, 2001).

Collectors traditionally summarize collection methods within narratives in notebooks and databases, but parsed data fields ensure that data are methodically and consistently collected. Suggested sampling fields that document observed population structure and collection strategies are included in Table 2.

\section{CONCLUSIONS}

It is becoming clear that many future improvements in agriculture will rely on novel genes and gene complexes provided by wild genetic resources (Tanksley and McCouch, 1997). Genomic efforts have begun to mine key wild individuals for novel alleles that could be used in crop improvement efforts (Tanksley and McCouch, 1997). Subsets of collections that capture most of the alleles (allele-mining sets) have been specifically designed for this purpose (Zhao et al., 2010). Many current efforts are focused on traits controlled by relatively few genes identified within quantitative trait loci. These genes can be taken from a wild background and inserted into a desirable cultivar using transgenic or selective breeding techniques. With more focus on using genes of similar plant origin for crop improvements (rather than interspecific transgenics), identifying desirable alleles of key genes within plants is critical.

Placing functional variation into a spatial context is an approach that can lead to a more complete understanding of genes that result in adaptation and, importantly, can serve as a rational method for identifying natural variation of potential use for agriculture (Fig. 1). With detailed inputs, ecological niche modeling analyses can target new collection regions and novel forms of adaptation (RamírezVillegas et al., 2010). For example, germplasm collection prioritizations have been established based on predictive modeling using known geographic distribution, habitat, and climate data. Collectors successfully identified

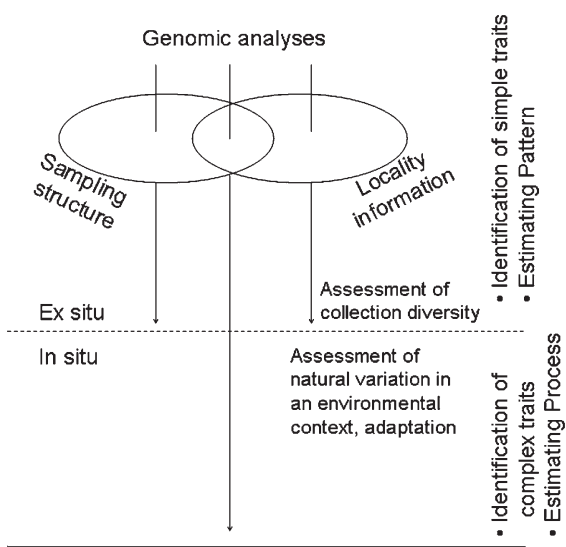

Fig. 1. The availability of detailed genomic, sampling, and locality information for genebank accessions allows collections to be evaluated for more than simple traits and patterns. The data intersection facilitates assessments of national variation, which is key for identifying complex traits and estimating processes.

novel wild pepper (Capsicum flexuosum) populations based on the model's predictions (Jarvis et al., 2005).

Combining a mechanistic understanding of gene function with a population genetic description of pattern and history will make it possible to understand the origins and age of alleles that influence fitness in the wild. New approaches that use natural variation for agricultural crop improvement will greatly enhance our knowledge of genetic adaptation in the wild (González-Martínez et al., 2007; Pauwels et al., 2008; Stapley et al., 2010; Travers et al., 2007). The challenge will hinge on the quality of data associated with wild accessions. To enable this integrative approach, accurate passport, habitat, and sampling data must be available. Without data standards in these areas, research using variation within the collection holdings will not be able to take advantage of emerging approaches that will lead to more efficient allele-mining efforts and management practices.

\section{Literature Cited}

Antonovics, J. 1976. The input from population genetics: "The new ecological genetics." Syst. Bot. 1:233-245.

Bhullar, N.K., K. Street, M. Mackay, N. Yahlaoul, and B. Keller. 2009. Unlocking wheat genetic resources for the molecular identification of previously undescribed functional alleles at the Pm3 resistance locus. Proc. Natl. Acad. Sci. USA 106:9519-9524.

Bishop, G.J., K. Harrison, and J.D.G. Jones. 1996. The tomato Dwarf gene isolated by heterologous transposon tagging encodes the first member of a new cytochrome P450 family. Plant Cell 8:959-969.

Black, W.C., C.F. Baer, M.F. Antolin, and N.M. DuTeau. 2001. Population genomics: Genomewide sampling of insect populations. Annu. Rev. Entomol. 46:441-469.

Caicedo, A.L. 2008. Geographic diversity cline of $R$ gene homologs in wild populations of Solanum pimpinellifolium. Amer. J. Bot. 95:393-398.

Chapman, M.A., C.H. Pashley, J. Wenzler, J. Hvala, S. Tang, S.J. Knapp, and J.M. Burke. 2008. A 
genomic scan for selection reveals candidates for genes involved in the evolution of cultivated sunflower (Helianthus annuus). Plant Cell 20: 2931-2945.

Darwin Core Task Group. 2011. Darwin Core. 28 Feb. 2011. <http://rs.tdwg.org/dwc/index.htm>.

Day Rubenstein, K., M. Smale, and M.P. Widrlechner. 2006. Demand for genetic resources and the U.S. National Plant Germplasm System. Crop Sci. 46:1021-1031.

Edwards, J.L., M.A. Lane, and E.S. Nielsen. 2000. Interoperability of biodiversity databases: Biodiversity information on every desktop. Science 289:2312-2314.

González-Martínez, S.C., N.C. Wheeler, E. Ersoz, C.D. Nelson, and D.B. Neale. 2007. Association genetics in Pinus taeda L. I. Wood property traits. Genetics 175:399-409.

Greene, S.L. and G.A. Pederson. 1996. Eliminating duplicates in germplasm collections: A white clover example. Crop Sci. 36:1398-1400.

Gur, A. and D. Zamir. 2004. Unused natural variation can lift yield barriers in plant breeding. PLoS Biol. 2:e245.

Hajjar, R. and T. Hodgkin. 2007. The use of wild relatives in crop improvement: A survey of developments over the last 20 years. Euphytica 156:1-13.

Hijmans, R.J., S.E. Cameron, J.L. Parra, P.G. Jones, and A. Jarvis. 2005. Very high resolution interpolated climate surfaces for global land areas. Intl. J. Climatol. 25:1965-1978.

Hijmans, R.J., M. Schreuder, J. De la Cruz, and L. Guarino. 1999. Using GIS to check coordinates of genebank accessions. Genet. Resources Crop Evol. 46:291-296.

Hill, A.W., R. Guralnick, P. Flemons, R. Beaman, J. Wieczorek, A. Ranipeta, V. Chavan, and D. Remsen. 2009. Location, location, location: Utilizing pipelines and services to more effectively georeference the world's biodiversity data. BMC Bioinformatics, doi: 10.1186/14712105-10-S14-S3.

Holbrook, C.C., W.F. Anderson, and R.N. Pittman. 1993. Selection of a core collection from the U.S. germplasm collection of peanut. Crop Sci. 33:859-861.

Hyten, D.L., Q. Song, Y. Zhu, I.-K. Choi, R.L. Nelson, J.M. Costa, J.E. Specht, R.C. Shoemaker, and P.B. Cregan. 2006. Impacts of genetic bottlenecks on soybean genome diversity. Proc. Natl. Acad. Sci. USA 103:16666-16671.

Jarvis, A., K. Williams, D. Williams, L. Guarino, P.J. Caballero, and G. Mottram. 2005. Use of GIS for optimizing a collecting mission for a rare wild pepper (Capsicum flexuosum Sendtn.) in Paraguay. Genet. Resources Crop Evol. 52:671-682.
Johal, G.S., P. Balint-Kurti, and C.F. Weil. 2008. Mining and harnessing natural variation: A little MAGIC. Crop Sci. 48:2066-2073.

Kaur, N., K. Street, M. Mackay, N. Yahiaoui, and B. Keller. 2008. Molecular approaches for characterization and use of natural disease resistance in wheat. Eur. J. Plant Pathol. 121:387-397.

Kelling, S. 2008. Significance of organism observations: Data discovery and access in biodiversity research. Report for the Global Biodiversity Information Facility, Copenhagen, Denmark.

Kennedy, J., R. Hyam, R. Kukla, and T. Paterson. 2006. Standard data model representation for taxonomic information. OMICS 10:220 230.

Khoury, C., B. Laliberté, and L. Guarino. 2010. Trends in ex situ conservation of plant genetic resources: A review of global crop and regional conservation strategies. Genet. Resources Crop Evol. 57:625-639.

Kumar, G.R., K. Sakthivel, R.M. Sundaram, C.N. Neeraja, S.M. Balachandran, N.S. Rani, B.C Viraktamath, and M.S. Madhav. 2010. Allele mining in crops: Prospects and potentials. Biotechnol. Adv. 28:451-461.

Latha, R., L. Rubia, J. Bennett, and M.S. Swaminathan. 2004. Allele mining for stress tolerance genes in Oryza species and related germplasm. Mol. Biotechnol. 27:101-108.

Luikart, G., P.R. England, D. Tallmon, S. Jordan, and P. Taberlet. 2003. The power and promise of population genomics: From genotyping to genome typing. Natl. Rev. 4:981-994.

Manel, S., M.K. Schwartz, G. Luikart, and P. Taberlet. 2003. Landscape genetics: Combining landscape ecology and population genetics. Trends Ecol. Evol. 18:189-197.

Mardis, E.R. 2008. The impact of next-generation sequencing technology on genetics. Trends Genet. 24:133.

McCouch, S. 2004. Diversifying selection in plant breeding. PLoS Biol. 2:e347.

Pauwels, M., N. Roosens, H. Frérot, and P. SaumitouLaprade. 2008. When population genetics serves genomics: Putting adaptation back in a spatial and historical context. Curr. Opin. Plant Biol. 11:129-134.

Ramírez-Villegas, J., C. Khoury, A. Jarvis, D.G. Debouck, and L. Guarino. 2010. A gap analysis methodology for collecting crop genepools: A case study with Phaseolus beans. PLoS One 5:E13497.

Schoen, D.J. and A.H.D. Brown. 1993. Conservation of allelic richness in wild crop relatives is aided by assessment of genetic markers.
Proc. Natl. Acad. Sci. USA 90:1062310627.

Schoen, D.J. and A.H.D. Brown. 2001. The conservation of wild plant species in seed banks. Bioscience 51:960-966.

Stapley, J., J. Reger, P.G.D. Feulner, C. Smadja, J. Galindo, R. Ekblom, C. Bennison, A.D. Ball, A.P. Beckerman, and J. Slate. 2010. Adaptation genomics: The next generation. Trends Ecol. Evol. 25:705-712.

Steiner, J.J. and S.L. Greene. 1996. Proposed ecological descriptors and their utility for plant germplasm collections. Crop Sci. 36: 439-451.

Tanksley, S.D. and S.R. McCouch. 1997. Seed banks and molecular maps: Unlocking genetic potential from the wild. Science 277:10631066.

Taxonomic Data Working Group. 2011. 28 Feb. 2011. <http:www.tdwg.org/standards/>.

Travers, S.E., M.D. Smith, J. Bai, S.H. Hulbert, J.E. Leach, P.S. Schnable, A.K. Knapp, G.A. Milliken, P.A. Fay, A. Saleh, and K.A. Garrett. 2007. Ecological genomics: Making the leap from model systems in the lab to native populations in the field. Front. Ecol. Environ 5:19-24.

Vigouroux, Y., M. McMullen, C.T. Hittinger, K. Houchins, L. Schulz, S. Kresovich, Y. Matsuoka, and J. Doebley. 2002. Identifying genes of agronomic importance in maize by screening microsatellites for evidence of selection during domestication. Proc. Natl. Acad. Sci. USA 99:9650-9655.

Walters, C., G.M. Volk, and C.M. Richards. 2008. Genebanks in the post-genomic age: Emerging roles and anticipated uses. Biodiversity 9: 68-71.

Wang, J.-C., J. Hu, N.-N. Liu, H.-M. Xu, and S. Zhang. 2006. Investigation of combining plant genotypic values and molecular marker information for constructing core subsets. J. Integr. Plant Biol. 48:1371-1378.

Williams, K.A. 2005. An overview of the U.S. National Plant Germplasm System's exploration program. HortScience 40:297-301.

Xiao, J., J. Li, L. Yuan, and S.D. Tanksley. 1996. Identification of QTLs affecting traits of agronomic importance in a recombinant inbred population derived from a subspecific rice cross. Theor. Appl. Genet. 92:230-244.

Zhao, W., G.-T. Cho, K.-H. Ma, J.-W. Chung, J.-G. Gwag, and Y.J. Park. 2010. Develoment of an allele-mining set in rice using a heuristic algorithm and SSR genotype data with least redundancy for the post-genomic era. Mol. Breed. 26:639-651. 